\title{
Detection of High Level Aminoglycoside Resistance and Vancomycin Resistance in Enterococcus Species Isolated from Various Clinical Samples of Tertiary Care Medical College Hospital
}

\author{
S. Rajesh ${ }^{*}$, N. Subathra ${ }^{2}$, D.Neelaveni ${ }^{3}$ and S. Nirmala ${ }^{4}$ \\ Department of Microbiology, Government Mohankumaramangalam Medical College, \\ Salem, India \\ *Corresponding author:
}

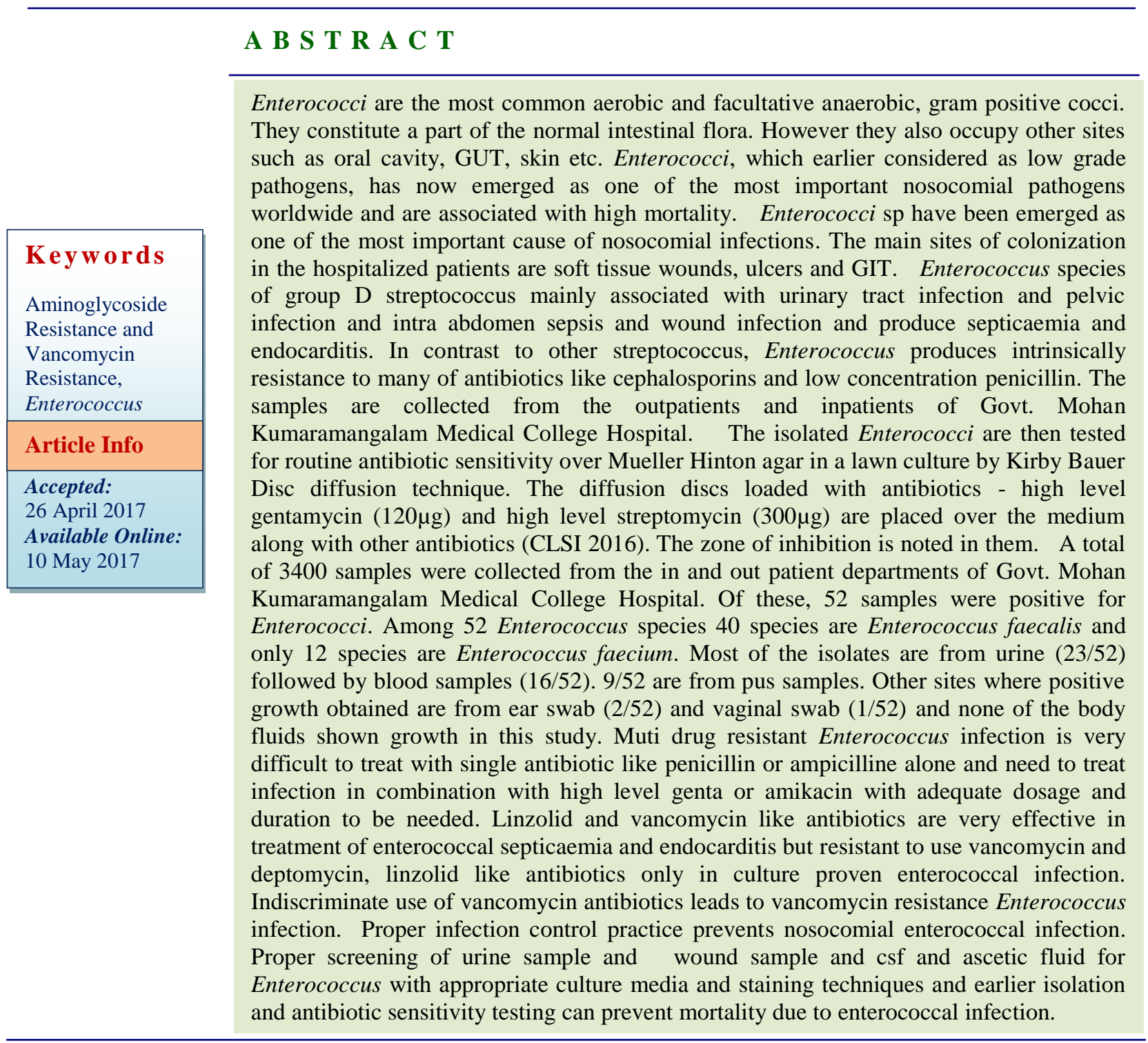




\section{Introduction}

Enterococci are the most common aerobic and facultative anaerobic, gram positive cocci. They constitute a part of the normal intestinal flora. However they also occupy other sites such as oral cavity, GUT, skin etc. Enterococci, which earlier considered as low grade pathogens, has now emerged as one of the most important nosocomial pathogens worldwide and are associated with high mortality.

The main sites of colonization in the hospitalized patients are soft tissue wounds, ulcers and GIT. Over the years the Enterococci have become increasingly resistant to antibiotics in terms of both multiplicity of resistance and level of resistance to a particular drug (Sieńkoe et al., 2014; Triveda and Gomathi, 2016; Luna Athigari, 2010). Enterococcus species of group D Streptococcus mainly associated with urinary tract infection and pelvic infection and intra abdomen sepsis and wound infection and produce septicaemia and endocarditis.

In contrast to other Streptococcus, Enterococcus produces intrinsically resistance to many of antibiotics like cephalosporins and low concentration penicillin like cell wall active antibiotics which are mainly used for treatment of other Streptococcus infection. Enterococcus is also resistance to low concentration aminoglycoside like amikacin and gentamycin and shows sensitive only to high level of aminoglycosides. Enterococcus extrinsically resistant to high concentration of ampicilline and high level aminoglycosides and ciprofloxacin due to betalactamase enzyme production and plasmid mediated gene transfer mechanisms and through transporans. Due to multi drug resistance Enterococcus infections are very difficult to treat. Enterococci are intrinsically resistant to cell wall active agents like penicillin, Ampicillin if used alone. They are also inherently or intrinsically resistant to other antibiotics such as Cephalosporins and Aminoglycosides. This type of resistance is due to loss of affinity to PBPs in case of cell wall active agents and reduced uptake of antibiotic as in case of AGs.

If any of those antibiotics when used alone, resulting in treatment failure. To overcome these resistance, various combination therapies have been used combining an Aminoglycoside antibiotic with one of the cell wall active agents. These combination leads to synergism known as enhanced killing of organism by the drugs (Gangurde et al., 2014).

The common regime for treatment for serious Enterococcus infections such as septicemia is combination of cell wall inhibitors such as penicillin, ampicillin or vancomycin with aminoglycosides such as gentamicin and Amikacin.

The addition of cell wall inhibitor agents helps in penetration of aminoglycosides into the bacterial cytoplasm making intrinsically resistant organism as aminoglycoside sensitive (Wei Jia et al., 2014). This study will provide data on the prevalence of High Level Aminoglycoside Resistance among Enterococci species and determine the usefulness of combination therapy with cell wall active agents to treat serious Enterococcal infections like meningitis, endocarditis and septicemia

The main aim and objectives of this study includes, isolating and identifying the Enterococcus bacteria from various clinical samples and detecting the High Level Aminoglycoside Resistance (HLAR) among Enterococcus isolates. Also to assess the usefulness of combination therapy of Aminoglycosides with cell wall active antibiotics to treat serious enterococcal infections. 
Materials and Methods

\section{Methods of data collection}

Study design and type: Prospective study

Study population: Male and Female patients of all age groups from various in and outpatient departments

Place of Study: Department of Diagnostic Microbiology, Govt. Mohan Kumaramangalam Medical College, Salem District, Tamilnadu

Period of study: Six months (June 2016 November 2016)

Sample size: 3400

Sample Selection criteria: All pus samples, urine samples, blood samples, body fluids (Pleural, Peritoneal) and CSF

Sample Exclusion Criteria: samples from respiratory system and from GI system as Enterococci occur as normal commensals in these sites

\section{Methodology}

The samples are collected from the outpatients and inpatients of Govt. Mohan Kumaramangalam Medical College Hospital. The samples are cultured over basal medium nutrient agar, McConkey agar without crystal violet and enriched medium such as blood agar. The Enterococcus sp. are identified by Gram staining morphology, 3\% catalase test and confirmed by Salt tolerance test (growth in $6.5 \% \mathrm{Nacl}$ ), Bile aesculin hydrolysis and heat test. Speciation of Enterococci were carried out by Facklam's and Collin's conventional method. Growth of black colonies on $0.04 \%$ Potassium tellurite agar and fermentation of sorbitol, mannitol sugars but not arabinose were identified as
E.faecalis. Fermentation of arabinose but not sorbitol without potassium tellurite reduction was identified as E. faecium (Facklam and Collins, 1989). The isolated Enterococci are then tested for routine antibiotic sensitivity over Mueller Hinton agar in a lawn culture by Kirby Bauer Disc diffusion technique. The diffusion discs loaded with antibiotics - high level gentamycin $(120 \mu \mathrm{g})$ and high level streptomycin $(300 \mu \mathrm{g})$ are placed over the medium along with other antibiotics (CLSI 2016). The zone of inhibition is noted in them. For HLAR, the resistance is indicated as no zone and susceptibility as zone of diameter greater than $10 \mathrm{~mm}$. Strains with inhibition $7-9 \mathrm{~mm}$ are considered as inconsistent. All media and antibiotic discs are purchased from Hi Media Laboratories, Mumbai.

\section{Results and Discussion}

A total of 3400 samples were collected from the in and out patient departments of Govt. Mohan kumaramangalam Medical College Hospital. Of these, 52 samples were positive for Enterococci. Among 52 Enterococcus species 40 species are Enterococcus faecalis and only 12 species are Enterococcus faecium (Table.3). Most of the isolates are from urine (23/52) followed by blood samples (16/52). 9/52 are from pus samples. Other sites where positive growth obtained are from ear swab $(2 / 52)$ and vaginal swab (1/52). None of the body fluids showed growth in this study (0/52) (Table 1).

All 52 isolates were screened for HLG and HLS resistance. 7/52 strains showed High Level Aminoglycoside resistance. Of which 4 showed only HLG resistance; HLS resistance is observed in 2 isolates and one isolate shows resistance to both (Table 2).

Table 3 shows general antibiotic sensitivity pattern of enterococcal isolates in which 
highest sensitivity is shown by linezolid (51/52) followed by vancomycin (47/52). The sensitivity of Amoxy-clauv and Ampicillin is around $40 \%$. For urinary isolates nitrofurantoin shows highest (96\%) sensitivity and sensitivity of Norfloxacin is poor $(25 \%)$. Doxycycline shows good spectrum of action (85\%) when compared to Ampicillin, Amoxyclav and erythromycin.

The Enterococcus bacteria are inherently resistant to the antibiotics in regular usage such as Low level aminoglycosides and cell wall inhibitors. Hence it is a challenging task to handle the Enterococcus species.

In most of the studies in India (Niharika et al., 2014; Jyotsna Agarwal et al., 2009), the maximum number of isolates were recovered from urine samples, blood samples followed by pus swab. The present study also showed that maximum number of isolates were from urine samples (44\%) followed by blood samples (31\%) and pus swabs (17\%).

In our study maximum isolate from urine (44\%) and blood(31\%) but Enterococcus isolation in blood is more in number as compared to other study (Sieńkoe et al., 2014) and Mendiratta et al., 2008). In contrast to most of the recent studies conducted in India (Narayan srihari et al., 2011; Niharika et al., 2014), The HLAR resistance pattern in the present study is low, only $14 \%$ when compared $(50-90 \%$ in other studies), The HLGR alone is $8 \%$, HLSR alone is $4 \%$ and $2 \%$ show both type resistances simultaneously. In a study comprising 27 European countries by Schoutan et al.,(1999) the HLGR prevalence rate varied from1$49 \%$.high level gentamycin resistance also is very less (14\%) as compared to other study (Sivasankari et al., 2013)

In our study shows, more number of 40 Enterococcus faecalis (76.9\%) as compared to 12 Enterococcus faecium (23.1\%). it is similar to other study like Gangurde et al., (2014) and Latika et al., (2012). But some of studies shows more number of Enterococcus faecium and less number of Enterococcus faecalis (Wei Jia et al., 2014) Ampicillin resistance is only $33 \%$ as compared to other study (Mitrakhani et al., 2016) it is very less in number only. More than $50 \%$ of isolates are resistant to Ampicillin, Amoxyclav, ciprofloxacin and Erythromycin in the present study. This is comparable with other recent studies (Narayan Srihari et al., 2011, Adhikari et al., 2010).

Table.1 Percentage of Enterococcus isolates

\begin{tabular}{|l|c|c|}
\hline Samples & Enterococci Isolated & Percentage (\%) \\
\hline Pus & 9 & $17.3 \%$ \\
Sputum & 1 & $2.0 \%$ \\
Blood & 16 & $\mathbf{3 0 . 8 \%}$ \\
Urine & 23 & $44.2 \%$ \\
Ear swab & 2 & $4.0 \%$ \\
Vaginal swab & 1 & $2.0 \%$ \\
Body fluids* & 0 & 0 \\
\hline Total & 52 & \\
\hline
\end{tabular}

*Denotes CSF, Pleural and Peritoneal fluids 
Table.2 High Level Aminoglycoside Resistance among Enterococci

\begin{tabular}{|l|c|c|}
\hline Drug & Resistance $(\mathrm{n}=52)$ & Percent \\
\hline High Level Gentamycin $(120 \mu \mathrm{g})$ & $\mathbf{4}$ & $\mathbf{8 \%}$ \\
\hline High Level Streptomycin $(300 \mu \mathrm{g})$ & $\mathbf{2}$ & $\mathbf{4 \%}$ \\
\hline Both HLG and HLS & $\mathbf{1}$ & $\mathbf{2 \%}$ \\
\hline
\end{tabular}

Table.3 Distribution of enterococcus species

\begin{tabular}{|l|l|l|}
\hline Species identified & Number $(\mathrm{n}=52)$ & Percentage \\
\hline E. faecalis & $\mathbf{4 0}$ & $\mathbf{7 6 . 9} \%$ \\
\hline E. faecium & $\mathbf{1 2}$ & $\mathbf{2 3 . 1 \%}$ \\
\hline Total & $\mathbf{5 2}$ & $\mathbf{1 0 0} \%$ \\
\hline
\end{tabular}

Table.4 Antibiotic Sensitivity Pattern of Enterococcus to other antibiotics

\begin{tabular}{|c|c|c|}
\hline Antibiotic used & $\begin{array}{l}\text { Sensitive (\%) } \\
\mathbf{N}=52\end{array}$ & Resistant (\%) \\
\hline Ampicillin $(10 \mu \mathrm{g})$ & $19(36.5)$ & $33(63.5)$ \\
\hline Ciprofloxacin $(5 \mu \mathrm{g})$ & $29(55.8)$ & $23(44.2)$ \\
\hline Norfloxacin $^{\#}(\mathbf{n}=\mathbf{2 3})$ & $6(26)$ & $17(74)$ \\
\hline Nitrofurantoin* $(30 \mu \mathrm{g}) \quad(\mathbf{n}=\mathbf{2 3})$ & $22(95.7)$ & $1(4.3)$ \\
\hline Doxycycline $(30 \mu \mathrm{g})$ & $44(84.6)$ & $8(15.4)$ \\
\hline Amoxyclauv & $22(42.3)$ & $30(57.7)$ \\
\hline Erythromycin $(15 \mu \mathrm{g})$ & $24(46.2)$ & $28(53.8)$ \\
\hline Vancomycin $(30 \mu \mathrm{g})$ & $49(94.3)$ & $5(5.7)$ \\
\hline Linezolid $(30 \mu \mathrm{g})$ & $51(98)$ & $1(2)$ \\
\hline
\end{tabular}


Fig.1 Percentage of Enterococci in clinical samples

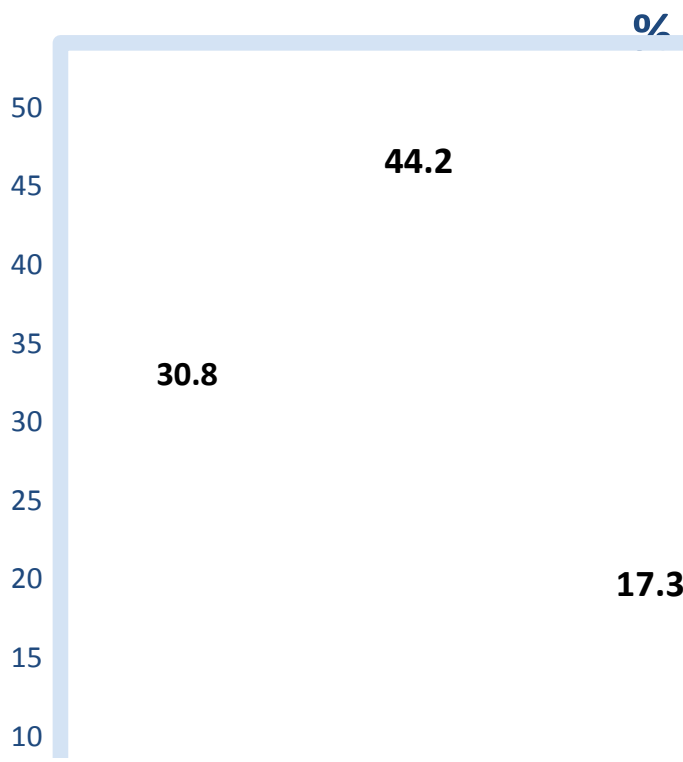

\% nE ENITEDnrnrrI

17.3

Fig.2 Distribution of High Level Aminoglycoside resistance

\% OF HLAR

2

4

8

According to our study the highest sensitivity pattern is shown by Linezolid (98\%) followed by vancomycin (94\%) for all samples and for urinary isolates nitrofurantoin shows high sensitivity (96\%) as observed in other studies. (Suresh et al., 2013; Agarwal et al., 2009; 1999).

Prevalence of Vancomycin Resistant Enterococci (VRE) by disk diffusion method varies about 0-6.5\% from various studies (Jyostna Agarwal et al., 2009). In the present study the incidence of VRE is $5.7 \%$ that is to be noted. Vancomycin resistance is only 5 $\%$.it is less as compared to Sieńkoe et al., (2014) study shows $24 \%$

Both Enterococcus faecalis and faecium shows equal range of drug resistance as compared to other study which shows more 
resistance seen only in faecium.

In conclusion, this study presents an antibiotic sensitivity pattern of Enterococcus isolates with special emphasis on prevalence of High Level Aminooglycoside Resistance (HLAR). According to present study, HLAR pattern is low (14\%) in our area of Salem District, Tamilnadu when compared to other parts of India.

Muti drug resistant Enterococcus infection is very difficult to treat with single antibiotic like pencillin or ampicilline alone and need to treat infection in combination with high level genta or amikacin with adequate dosage and duration to be needed. linzolid and vancomycin like antibiotics very effective in treatment of enterococcal septicaemia and endocarditis but restrict to use vancomycin and daptomycin, linzolid like antibiotics only in culture proven enterococcal infection. Indiscriminate use of vancomycin antibiotics leads to vancomycin resistance Enterococcus infection.

Proper infection control practice prevent nosocomial enterococcal infection. Proper screening of urine sample and wound sample and csf and ascetic fluid for Enterococcus with appropriate culture media and staining techniques and earlier isolation and antibiotic sensitivity testing can prevent mortality due to enterococcal infection. In the present study the second most common enterococcal infection is neonatal septicaemia which is one of the serious enterococcal infections in which combination therapy of AGs and cell wall active antibiotics would definitely be yielding successful treatment outcomes. For treating serious enterococcal infections single drug therapy with Linezolid or vancomycin is very effective and Nitrofurantoin effective in urine samples and for wound infections doxycycline can be tried.

At the same time, even the incidence of
Vancomycin resistance is low $(5.7 \%)$ by disk diffusion method when compared to other studies which mandates routine screening of VRE in our set up.

The greater understanding of mechanisms of antibiotic action and resistance pattern offers a hope on development of new therapeutic targets and hence helps the physician as well as the patient in better treatment of emerging resistant isolates.

Enterococcus infections are mainly associated with nosocomial infection and increase incidence mainly due to indiscriminate use of broad spectrum antibiotics. Proper infection control practice need to prevent nosocomial origin of entrococci.

\section{References}

Agarwal V.A. Jain Y.I., Pathak A.A. Concomitant high level resistance to penicillin and aminoglycosides in Enterococci at Nagpur, Central India. Indian J Med Microbiol 1999;17: 857.

Bhat KG, Paul C, Bhat MG. High level aminoglycoside resistance in Enterococci isolated from hospitalized patients.Indian J Med Res 1997; 105: 198-9.9.

Facklam RR, Collins MD. Identification of Enterococcus species isolated from human infections by conventional test scheme J Clin Microbiol 1989; 24:731-4.

Gangurde N, Mane M, Phatale S. Prevalence of Multidrug Resistant Enterococci in a Tertiary Care Hospital in India: A Growing Threat. Open J. Med. Microbiol., 2014;4:11-15.

Jyotsna Agarwal, Rajkumar Kalyan, and Mastan Singh. High-Level Aminoglycoside Resisance in Enterococci in tertiary care hospitals. Jpn. J. Infect. Dis., 62, 158-159, 2009 
Latika et al., 2012. Pervalence of Enterococcus with high resistance level. Open journal of medical microbiology, Volume 2 Issue 1.

Luna Athigari. High-level Aminoglycoside Resistance and Reduced Susceptibility to Vancomycin in Nosocomial Enterococci. J Glob Infect Dis. 2010 Sep-Dec; 2(3): 231-235.

Marothi, Y.A., H Agnihotri, D Dubey. Enterococcal resistance - An overview. Indian Journal of Medical Microbiology, (2005) 23(4):214-9.

Mendiratta, D.K., Kaur, H., Deotale, V., Thamke, D.C., Narang, R. and Narang, P. (2008) Status of High Level Aminoglycoside Resistant Enterococcus Faecium and Enterococcus Faecalis in a Rural Hospital of Central India. Indian Journal of Medical Microbiology, 26, 369-371. http://dx.doi.org/ 10.4103/ 0255-0857.43582

Mundy LM, Sahm DF, Gilmore M. Relationship between Enterococcal virulence and antimicrobial resistance. Clin. Microbiol. Rev., 2000; 13: 51322.

Narayan Shrihari, Kumudini T.S, S.G. Karadesai and S.C. Metgud. Speciation of enterococcal isolates and antibiotic susceptibility test including high level aminoglycoside resistance and minimum inhibitory concentration for vancomycin. Int $\mathrm{J}$ Biol Med Res.2011; 2(4): 865-869.

Niharika Lall, Silpi Basak. High level aminoglycoside resistant Enterococcus species: A study. Int J Cur Res Rev. 2014; 06(03).

Purva Mathur, Arti Kapil, Rachna Chandra, Pratibha Sharma \& Bimal Das.Antimicrobial Resistance in Enterococcus fecalis in tertiary care hospital in North India.Indian J Med Res 118, July 2003, pp 25-28
Randhawa, V.S., L. Kapoor, V. Singh \& G. Mehta. Aminoglycoside resistance in Enterococci isolated from pediatric septicemia in tertiary care hospital. Indian J Med Res 119 (Suppl) May 2004, pp 77-79

Sarika Jain, Ashwani kumar, Bineeta kashyap and Iqbal $\mathrm{R}$ Kaur. Clinico epidemiological profile and High level Aminoglycoside resistance in enterococcal septicemia from a tertiary care hospital in Delhi. Int $\mathbf{J}$ of Applied and Basic Medical Research, Jul- Dec 2011; Vol 1, Issue 2.

Schouten MA, Voss A, Hoogkamp-Korstanje JAA and the European VRE study group. Antimicrobial susceptibility patterns of Enterococci causing infections of Europe. ANtimicrob Agents Chemother 1999; 43: 2542-6.

Seema Sood, Meenakshi Malhotra, B.K. Das \& Arti Kapil. Enterococcal infections and antimicrobial resistance. Indian $\mathrm{J}$ Med Res 128, August 2008, pp 111121

Sieńko A., Wieczorek P., Wieczorek A., Sacha P., Majewski P., Ojdana D., Michalska A., Tryniszewska E. 2014. Occurrence of high-level aminoglycoside resistance (HLAR) among Enterococcus species strains. Prog Health Sci., Vol 4,

Sivasankari et al., 2013. Prevalence of hlar Enterococcus in tertiary care hospital. J. Pharm. Biol. Sci., Vol 8.

Sivasankari S, Somasunder V M, Senthamarai S, Anitha C, Kumudhavalli MS and Suneel kumar reddy A. IOSR Journal of pharmacy and Biological Sciences 2013 Nov-Dec; Vol 8, Issue 11

Stevenson KB, Murray EW, Sarubbi FA. Enterococcal meningitis: Report of four cases and Review. J Clin Infect Dis. 1994; 18:233-239.

Suresh et al.,. Isolation, Speciation and determination of high level 
aminoglycoside resistance of Enterococci. National Journal of Lab Medicine.2013; vol 2 (1): 12-15.

Triveda, L., S. Gomathi. High Level Aminoglycoside Resistance in Enterococcus Species Isolated from Tertiary Care Hospital of South India An Update. IJHSR. 2016; 6(7): 144Wei Jia, Gang Li and Wen Wang. 2014. Prevalence and Antimicrobial Resistance of Enterococcus Species: A Hospital-Based Study in China. Int. J. Environ. Res. Public Health, 11(3), 3424-3442. doi:10.3390/ijerph110303 424.

147

\section{How to cite this article:}

Rajesh, S., N. Subathra, D. Neelavani and Nirmala, S. 2017. Detection of High Level Aminoglycoside Resistance and Vancomycin Resistance in Enterococcus Species Isolated from Various Clinical Samples of Tertiary Care Medical College Hospital. Int.J.Curr.Microbiol.App.Sci. 6(5): 2731-2739. doi: https://doi.org/10.20546/ijcmas.2017.605.306 